\title{
SPECTROSCOPIC OBSERVATIONS OF THE SOUTHERN OLD NOVAE CP PUP AND V841 OPH
}

\author{
A.Bianchini ${ }^{1}$, M.Friedjung ${ }^{2}$, F.Sabbadin ${ }^{1}$ \\ ${ }^{1}$ Osservatorio Astronomico, 35100-Padova, Italy \\ ${ }^{2}$ Institut d'Astrophysique, 98bis, Bd Arago, 75014 Paris, France
}

\section{Abstract}

New spectroscopic observations of the old nova CP Pup suggest most probable orbital period of 0.061375 days. First systematic monitoring of the very old nova V841 Oph reveals orbital motion with a most probable period of about 0.6 days. A slightly eccentric solution at $\mathrm{P}=0.3755$ days is less probable.

\section{Introduction}

Spectroscopic observations of the classical old novae CP Pup (1942) and V841 Oph (1848) were carried out in the periods 6-10 March 1988 and 16-20 March 1989 at the European Southern Observatory at La Silla, Chile. We used the $1.5 \mathrm{~m}$ ESO telescope equipped with the Boller and Chivens Cassegrain Spectrograph and the CCD detector. For CP Pup we obtained 49 spectra in 1988 and 56 spectra in 1989; for V841 Oph we obtained 15 spectra in 1988 and 44 spectra in 1989. The spectral range was 4000-5000 $\dot{A}$ with a dispersion of $60 \dot{A} / m m$. Exposure times were of $20 \mathrm{~min}$. All the spectra have been reduced with the standard IHAP software. Both the radial velocities and the equivalent widths of the $H \beta$ and the HeII $\lambda 4686$ emissions have been measured by fitting gaussians to the line profiles. Fourier analysis and the standard $\chi^{2}$ method were used for the search of periodic radial velocity variations.

\section{CP Pup (1044)}

The extremely short orbital period of this old nova has been discovered spectroscopically by Bianchini et al. (1985a,b) and, photometrically, by Warner (1985).

Using all the available radial velocities of 1982 (Duerbeck et al. 1987), 1984 (Bianchini et al. 1985a,b), 1988 and 1989 (this work), and considering averages of the $\mathrm{H} \beta$ and Hell measurements we obtain the following orbital parameters:

$$
\begin{gathered}
P=0.0613750\left( \pm 10^{-7}\right) \text { days } ; V_{\gamma}=10( \pm 1) K \mathrm{~m} / \mathrm{s} ; a \sin i=0.62( \pm 0.02) \times 10^{10} \mathrm{~cm} \\
e=0.0 ; T_{0}=2445322.0180( \pm 0.0001) \mathrm{JD}
\end{gathered}
$$

The fit to the data is shown in Fig. 1.

The $1988 \mathrm{HeII}$ radial velocities seem to behave differently from those of $1989 \mathrm{in}$ that they suggest the possible presence of other periodicities. The solution obtained by Duerbeck et al. (1987) cannot be excluded, because it almost coincides with one of the minor peaks of power spectrum ( $10 \%$ less probable). $\mathrm{H} \beta$ equivalent widths give a weakly probable solution at $\mathrm{P}=0.0630$ days. No eclipse effects are clearly seen. 


\section{V841 Oph (1848)}

The mean spectrum of V841 Oph shows the presence of a broad absorption around a central $\mathrm{H} \beta$ emission component.

Since Fourier analysis gives quite similar power spectra for both $\mathrm{H} \beta$ and the Hell $\lambda 4686$ cmission and the two radial velocity curves are practically indistinguishable, we decided to analyse the averages of the velocities derived from these two lines. The most probable period is 0.60422 days, though a second solution at $\mathrm{P}=0.37551$ cannot be totally excluded. The same result is obtained by applying the standard $\chi^{2}$ method. The solution at $\mathrm{P}=0.3755$ days gives an eccentric orbit with $c=0.1$. The best solution, with no eccentricity, is shown in Fig. 2. The orbital parameters are :

$$
\begin{gathered}
P=0.060423\left( \pm 10^{-6}\right) d a y s ; V_{\gamma}=-84( \pm 5) K m / s ; a \sin i=7.5( \pm 0.5) \times 10^{10} \mathrm{~cm} ; \\
e=0.0 ; T_{0}=2447226.100( \pm 0.001) J D
\end{gathered}
$$

Multivaried analysis suggests as most probable $P=0.59850$ days, also coinciding with one of the peaks of the power spectrum. Actually, this period perfectly fits all the 1989 data; 1988 data instead show considerable scatter. No clear indication of eclipses or periodic variations has been found in the equivalent widths of the lines.

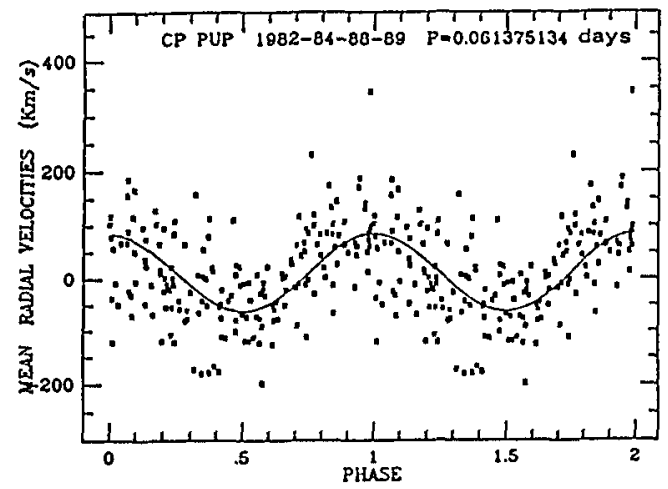

Fig.:1

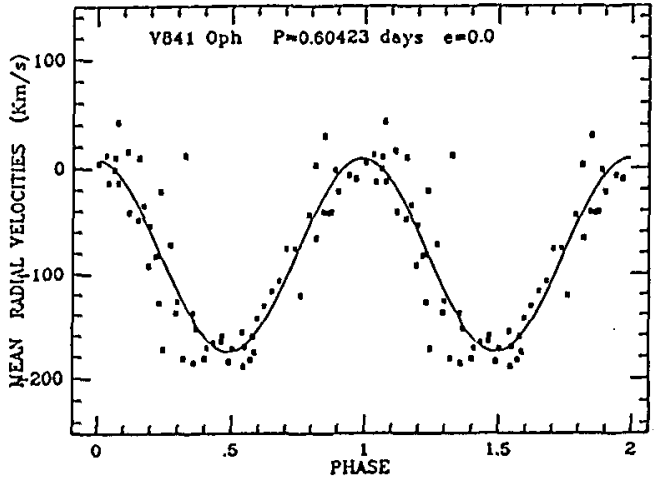

Fig.:2

\section{References}

- Bianchini,A.,Friedjung,M. and Sabbadin,F.,1985a.Inf.Bull.Var.Stars N.2650.

- Bianchini,A.,Friedjung,M. and Sabbadin,F.,1985b. In:Recent Results on Cataclysmic Variables, p.77, ESA SP-236.

- Duerbeck,H.W.,Seitter,W.S.and Duemmler, R.,1987. Mon. Not. R. astr. Soc. 229,653 .

- Warner,B.,1985. Mon. Not. R. astron. Soc. 217, 1P 